\title{
Neural Stem Cells Transplantation Promotes Brain Repair after Chronic Experimental Allergic Encephalomyelitis in Rat
}

\author{
Xiaoqing Gao1, Zhiqiang Mei², Yuanhui Du³, Li Deng1, Jie Du1* \\ ${ }^{1}$ Department of Anatomy and Neurobiology, Southwest Medical University, Luzhou, China \\ ${ }^{2}$ Preclinical Medicine Research Center, Southwest Medical University, Luzhou, China \\ ${ }^{3}$ Affiliated Hospital of Chinese Traditional Medicine, Sichuan Medical University, Luzhou, China \\ Email: *dujie75915@sohu.com
}

Received 8 April 2016; accepted 23 April 2016; published 27 April 2016

Copyright (C) 2016 by authors and OALib.

This work is licensed under the Creative Commons Attribution International License (CC BY). http://creativecommons.org/licenses/by/4.0/

(c) (i) Open Access

\begin{abstract}
The recent studies have showed neural stem cells (NSCs) transplantation promoted repair and functional recovery of chronic experimental allergic encephalomyelitis (EAE) animal model for MS based on the concept of cell replacement. In the present study, NSCs were transplantated into each lateral ventricle of rats at 10 days and sacrificed at 60 days after EAE immunization. We found that NSCs transplantation significantly reduced the clinical signs, suppressed brain inflammation and improved density of myelin. Transplanted NSCs differentiated into neurons and oligodendrocytes. Moreover, the number of endogenous neurons and oligodendrocytes in the rats treated with NSCs was significantly higher compared to the rats with saline. These results suggest an important neuroprotective role for NSCs that can potentially be exploited in cell-based therapeutic approaches for EAE.
\end{abstract}

\section{Keywords}

Chronic Experimental Allergic Encephalomyelitis, Multiple Sclerosis, Neural Stem Cells, Transplantation

\section{Subject Areas: Neuroscience}

\section{Introduction}

Multiple sclerosis (MS) is an autoimmune disease in the central nervous system (CNS) primarily affecting young adults, with a higher proportion of females [1] [2]. And MS is induced and mediated by myelin-reactive T cells

${ }^{*}$ Corresponding author.

How to cite this paper: Gao, X.Q., Mei, Z.Q., Du, Y.H., Deng, L. and Du, J. (2016) Neural Stem Cells Transplantation Promotes Brain Repair after Chronic Experimental Allergic Encephalomyelitis in Rat. Open Access Library Journal, 3: e2642.

http://dx.doi.org/10.4236/oalib.1102642 
responses against myelin antigens that results in progressive functional deficits [3]. In MS, myelin-reactive T cells migrate from peripheral tissues into the CNS where they are reactivated, thereby triggering an inflammatory cascade that leads to extensive loss of myelin and myelinating cells (oligodendrocytes) as well as damage to axons and neurons [4] [5]. Neural stem cells (NSCs) are immature cells with abilities to renew themselves and give rise to various neural cell types [6]. Moreover, NSCs have myelinogenic potential in the injured areas [7] and can attenuate the inflammatory brain process [8]. Therefore, NSCs has been proposed as a means of cell replacement therapy for experimental allergic encephalomyelitis (EAE), an animal model of MS. Here, we transplanted NSCs into lateral ventricle of rats subjected to chronic EAE and showed that NSCs had a profound effect on disease progression. More importantly, treatment with NSCs influenced neural cell fate in EAE host animals, promoting proliferation of neurons and oligodendrocytes. Based on these observations, we propose that NSCs represent a powerful cell based therapy for EAE or MS.

\section{Materials and Methods}

\subsection{Reagents and Instruments}

DMEM/F12 medium, basic fibroblast growth factor (bFGF), epidermal growth factor (EGF), and N2 supplement were from Gibco, USA. 5-bromo-2'-deoxyuridine (BrdU), complete Freund's adjuvant, Luxol fast blue (LFB) were from Sigma, USA. Mycobacterium tuberculosis H37Ra and bordetella pertusis toxin were from Shijiazhuang Weitian Scientific Instruments Equipment Co., Lid., China. Rabbit anti-microtubule-associated protein 2 (MAP2) and mouse anti-BrdU were from Abcam, UK. Rabbit anti-Galactocerebroside (GalC) was from Chemicon, USA. Goat anti-mouse IgG, goat anti-rabbit IgG, 5-Bromo-4-Chloro-3-Indolyl Phosphate/Nitroblue tetrazolium chloride (BCIP/NBT), alkaline phosphatase (AP)-streptavidin, horseradish peroxidase (HRP)-streptavidin, diaminobenzidine (DAB) and 3-amino-9-ethylcarbazole (AEC) were from Wuhan Boster Biological Technology, China. Stereotactic device (Angle Two ${ }^{\mathrm{TM}}$ Stereotaxic Instrument w/Rat Atlas Product: \#464601, USA).

\subsection{Preparation of NSCs}

The culture of NSCs was performed as described in previous study [9]. Briefly, the cell suspensions from the cerebral hemispheres of newborn Wistar rats (inbred strain, Animal House Center, Southwest Medical University, Sichuan, PR China) were placed in $25 \mathrm{ml}$ flask at a density of $1 \times 10^{5}$ cells/ml in serum-free DMEM/F12 medium supplemented with $20 \mathrm{ng} / \mathrm{ml}$ of bFGF, EGF and 1\% N2 supplement. After 5 - 7 days of culture, neurospheres were formed and assayed for nestin expression by immunohistochemical staining. For labeling the grafted cells in vivo, NSCs were pre-treated for 3 days before grafting with $10 \mu \mathrm{M}$ of BrdU.

\subsection{EAE Induction and NSCs Transplantation}

All animal experiments were approved by the Chinese Academy of Sciences, PR China. EAE is induced in female Wistar rats by guinea pig spinal cord homogenate emulsified with complete Freund's adjuvant. Briefly, Wistar rats (female, 6-8 week old, Southwest Medical University) were injected in four footpads with $100 \mathrm{mg}$ of guinea pig spinal cord tissue in $0.4 \mathrm{ml}$ of PBS emulsified with equal volume of CFA supplemented with $6 \mathrm{mg} / \mathrm{ml}$ of mycobacterium tuberculosis H37Ra. On the day of immunization and at day 2, $300 \mathrm{ng}$ of bordetella pertusis toxin in 0.1 ml PBS was injected subcutaneously. Rats were daily monitored for the severity of clinical disease. After immunization, the following scale for clinical symptoms was utilized: 0 . no clinical symptom; 1) limpness in tail; 2) hind-leg ataxia; 3) hind-leg paralysis; 4) Paraplegia; 5) moribund or dead. Higher neurological scores indicated more severe clinical symptoms. At ten days post-EAE induction, rats were anesthesized with intraperitoneal injection of pentobarbital sodium (30 mg/kg) and then were fixed in a stereotactic device. Quantities of $5 \times 10^{5}$ NSCs in a volume of $10 \mu \mathrm{l}$ were injected once into each lateral ventricle (bregma as origin, $\mathrm{AP}=-0.8$ to $1.0 \mathrm{~mm}$, $\mathrm{L}$ or $\mathrm{R}=-1.8$ to $2.0 \mathrm{~mm}, \mathrm{~V}=-4.0$ to $5.0 \mathrm{~mm}$ ). The control group experienced the same injection with $10 \mu \mathrm{l}$ saline into each lateral ventricle.

\subsection{Histological and Immunohistochemical Assessment}

Animals were sacrificed with a lethal dose of pentobarbital sodium at day 60 post-EAE induction. Ten rats (5 per 
group) were perfused by $4 \%$ paraformaldehyde for histopathological analysis. Histopathology and immunohistochemistry were assessed in $6 \mu \mathrm{m}$ thick paraffin sections at various levels (for bregma -0.2 to $-1.6 \mathrm{~mm}$ ). Hematoxylin and eosin (H and E) staining was used to evaluate inflammatory infiltration. Sections were stained with hematoxylin for $5 \mathrm{~min}$, rinsed with running water for $10 \mathrm{~min}$ and stained with $0.5 \%$ eosin for 30 seconds. Stained sections were then dehydrated in $95 \%$ alcohol and $100 \%$ alcohol, xylene transparency, and neutral gum mounting. 10 nuclei or more gathered in cerebrum white matter or surrounded by a blood vessel was regarded as an infiltration lesion. To estimate the degree of inflammation, the number of infiltrates and the number of cells per infiltrate were counted. LFB staining was used to observe myelin sheath and the measurement parameter was the integrated optical density (IOD). Sections were incubated in 0.1\% LFB dissolved in $95 \%$ ethanol and $0.05 \%$ acetic acid at $60^{\circ} \mathrm{C}$ for $24 \mathrm{~h}$. Stained sections were differentiated in $0.05 \%$ lithium carbonate for $10 \mathrm{sec}$ and $70 \%$ ethanol for $10 \mathrm{sec}$, then dehydrated and mounted.

BrdU immunohistochemistry staining was used to identify grafted cells in brain. Sections were incubated with mouse anti-BrdU (1:200) at $4^{\circ} \mathrm{C}$ overnight, then goat anti-mouse IgG (1:100) was added at $37^{\circ} \mathrm{C}$ for $30 \mathrm{~min}$. After AP-streptavidin was incubated at $37^{\circ} \mathrm{C}$ for $30 \mathrm{~min}$, BCIP/NBT was used as a chromogen for $10 \mathrm{~min}$. For double-labeling experiments, MAP2 and GalC were used to identify differentiated cells from grafted cells. Sections were incubated again with rabbit anti-MAP2 (1:100) and GalC (1:50) at $4^{\circ} \mathrm{C}$ overnight, goat anti-rabbit IgG (1:100) was then incubated for $30 \mathrm{~min}$. HRP-streptavidin was incubated for $30 \mathrm{~min}$, followed by DAB or AEC for $10 \mathrm{~min}$ as a chromogen. Immunohistochemistry controls were routinely performed with incubations in which primary antibodies were omitted.

Myelin sheath was assessed as IOD in corpus callosum in 3 sections per animal by ImageJ $1.44 \mathrm{p}$ software. The infiltrates in the corpus striatum were counted in sections per rats. And 3 fields/each striatum were imaged, so 6 fields in bilateral striatum were imaged and counted. Immunopositive cells were counted in 6 fields situated within bilaterally striatum in 3 sections per animal. Measurement was made in a pre-defined field $(0.6 \mathrm{~mm} \times 0.6$ $\mathrm{mm}$ ) by image-pro plus 6.0 software.

\subsection{Statistical Analysis}

All measured values were expressed as mean \pm SD, and statistical analysis was performed using SPSS software (17.0). For the clinical scores, the multiple comparisons were done using two-way repeated measures ANOVA followed by Tukey's post test for multiple pair-wise examinations. For the other histologicaland immunostaining analysis, multiple comparisons were done using one-way ANOVA followed by Tukey's post hoc test for multiple pair-wise examinations. Difference was considered significant at $P<0.05$.

\section{Results}

\subsection{Culture of NSCs}

The cells isolated from newborn rats cerebral hemispheres were proliferated into round floating spheres after 5 - 7 days of culture (Figure 1(a)), which expressed the neuroepithelial cells specific markers nestin (Figure 1(b)).

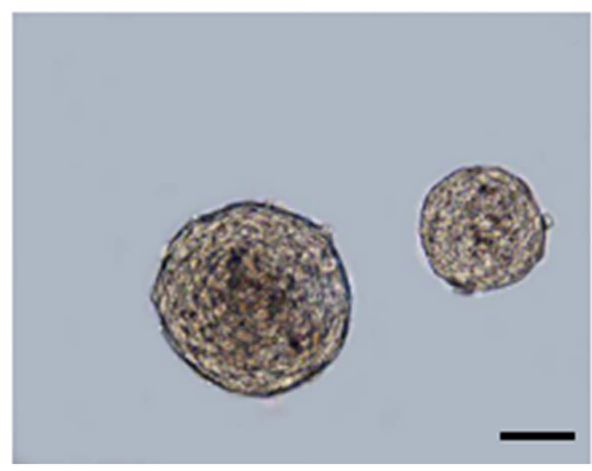

(a)

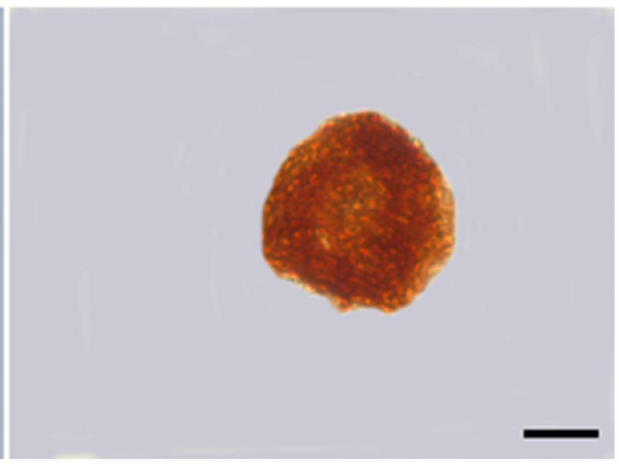

(b)

Figure 1. The generation and characterization of NSCs in vitro. (a) Cultured neurospheres derived from cerebral tissue of newborn rats at culture of $7 \mathrm{~d}$. (b) Immunocytochemical staining of nestin in neurospheres. Bar $=50 \mu \mathrm{m}$. 


\subsection{Attenuation of Clinical Symptoms Following NSCs Transplantation}

To evaluate the effects of NSCs on EAE rats, NSCs were transplanted into lateral ventricles before the onset of the disease (on day 10 after EAE induction). Disabilities appeared at 11 - 12 days after the initial EAE induction. As shown in Figure 2, the rats receiving NSCs had reduced clinical symptoms compared to the control rats $(P<0.05)$. Although the control rats showed improved clinical scores over time, there were apparent deficits at the end of the follow-up period (60 days post-EAE induction). The rats that received NSCs recovered their normal gait at 50 days post-EAE induction.

\subsection{Attenuated Inflammation and More Myelin Sheath by NSCs Transplantation}

Inflammatory infiltrates and density of myelin sheath in brain of EAE rats were evaluated at 60 days after EAE-induction. The majority of infiltrates were located in the corpus striatum adjacent to fibers and the rats with saline showed more infiltrates than the rats with transplantation $(P<0.05)$ (Figures 3(a)-(c)), moreover, fewer cells in per infiltration were observed in rats NSCs compared with rats with saline $(P<0.05)$ (Figure 3(d)). The saline-injected rats showed sparse and thin myelin sheath (Figure 3(e)). NSCs transplantation significantly increased the density of myelin sheath shown by stronger color intensity than did control rats $(P<0.05)$ (Figures $3(\mathrm{f})-(\mathrm{g}))$.

\subsection{NSCs Transplantation Promoted the Proliferation of Neurons and Oligodendrocytes}

The transplanted cells were detected by BrdU-immunostaining in rats at 60 days after EAE-induction. The BrdU ${ }^{+}$ cells located predominantly in the inflamed areas (Figure 4(a) and Figure 4(b), solid arrow). The BrdU ${ }^{+}$cells differentiated into neurons (Figure 4(a), arrowhead) and oligodendrocytes (Figure 4(b), arrowhead). To observe the effect of NSCs transplantation on the proliferation of endogenous neurons and oligodendrocytes, the number of $\mathrm{MAP2}^{+} / \mathrm{BrdU}^{-}$(Figure 4(a), dotted arrow) and $\mathrm{GalC}^{+} / \mathrm{BrdU}^{-}$(Figure 4(b), dotted arrow) cells in the NSCs group were counted, and the MAP2 ${ }^{+}$(Figure 4(c), dotted arrow) and $\mathrm{GalC}^{+}$(Figure 4(d), dotted arrow) cells in the saline-injected group were also counted as controls. The number of endogenous neurons and oligodendrocytes per $\mathrm{mm}^{2}$ in rats with NSCs was significantly higher than that in rats with saline $(P<0.05$, Figure $4(\mathrm{e})$ ). Therefore, NSCs transplantation not only added exogenous neurons and oligodendrocytes by differentiation, but also promotes the proliferation of endogenous neurons and oligodendrocytes.

\section{Discussion}

Our study showed that grafting NSCs into rats subjected to EAE significantly improved function compared with control group. More myelin sheath and fewer inflammatory infiltrations were observed in NSCs group. Transplanted NSCs differentiated into neurons and oligodendrocytes. And more endogenous neurons and oligodendrocytes were observed in the NSCs group than those in the saline-injected group.

Studies have reported that NSCs transplantation could reduce the number of EAE-induced peripheral and CNS immune cells, and promote clinical improvement [10]-[12]. Injected subcutaneously NSCs migrated into lymph nodes of EAE mice where they hampered the activation of myeloid dendritic cells and steadily restrained

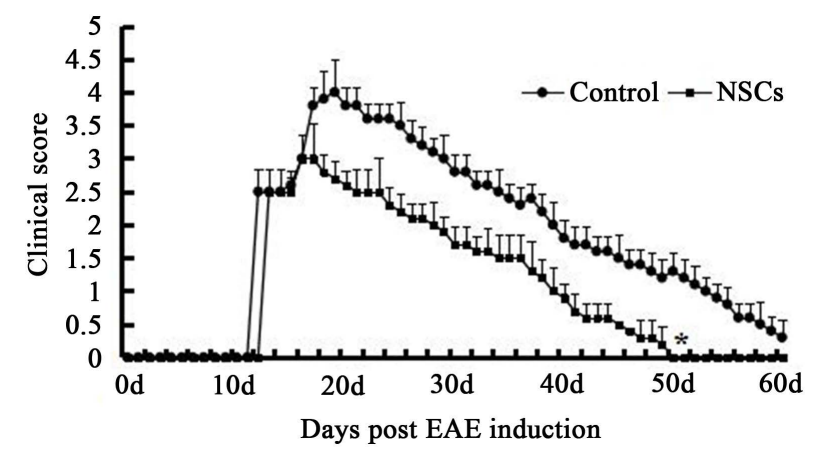

Figure 2. The improvement of clinical symptoms after NSCs transplantation (mean \pm SD, $\mathrm{n}=$ 5). ${ }^{*} P<0.05$ versus control group. 


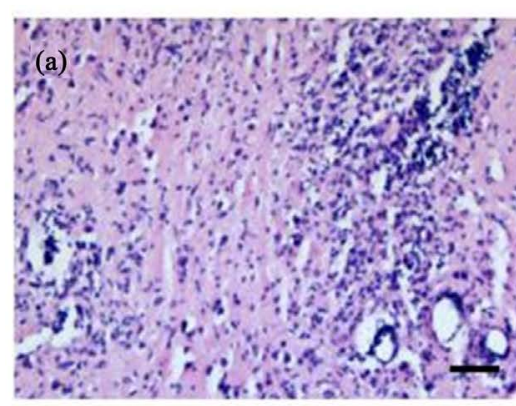

(e)

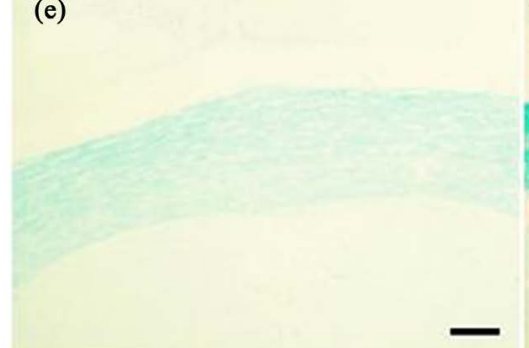

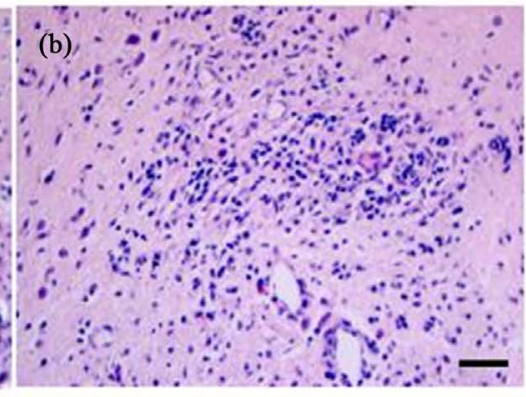

(f)

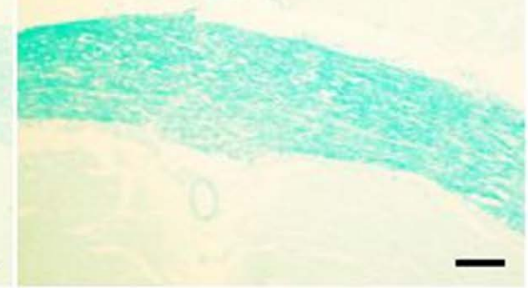

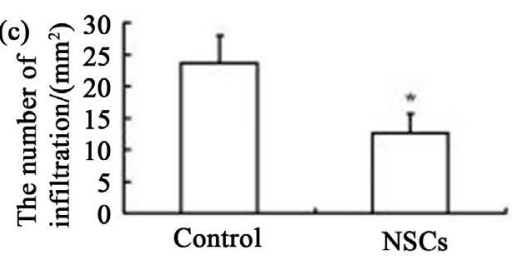

(d)

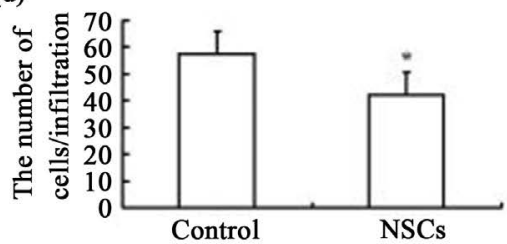

(g)

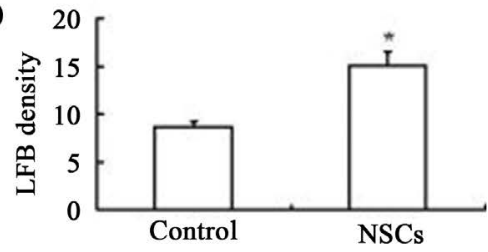

Figure 3. Attenuated inflammation and more myelin sheath after NSCs transplantationin. (a), (b) HE staining showed inflammatory infiltrates of the cerebral parenchyma in control and NSCs groups, respectively. The control group showed much more infiltration with inflammatory cells. The NSCs transplanted groups displayed a reduction in the number and size of the inflammatory infiltrations; (c) Quantitative analysis of the number of infiltrations per $\mathrm{mm}^{2}$ (mean $\pm \mathrm{SD}, \mathrm{n}=5$ ), ${ }^{*} P<0.05$ versus control group; (d) Quantitative analysis of the number of cells per infiltration (mean $\pm \mathrm{SD}, \mathrm{n}=5$ ), ${ }^{*} P<0.05$ versus control group; (e)-(f) LFB staining showed myelin sheath of the corpus callosum in control and NSCs groups respectively. Control group showed marked sparser and thinner myelin sheath compared with NSCs groups. (G) Quantitative analysis of LFB density (mean $\pm \mathrm{SD}, \mathrm{n}=5$ ). ${ }^{*} P<0.05$ versus control group. $\mathrm{Bar}=100 \mu \mathrm{m}$.

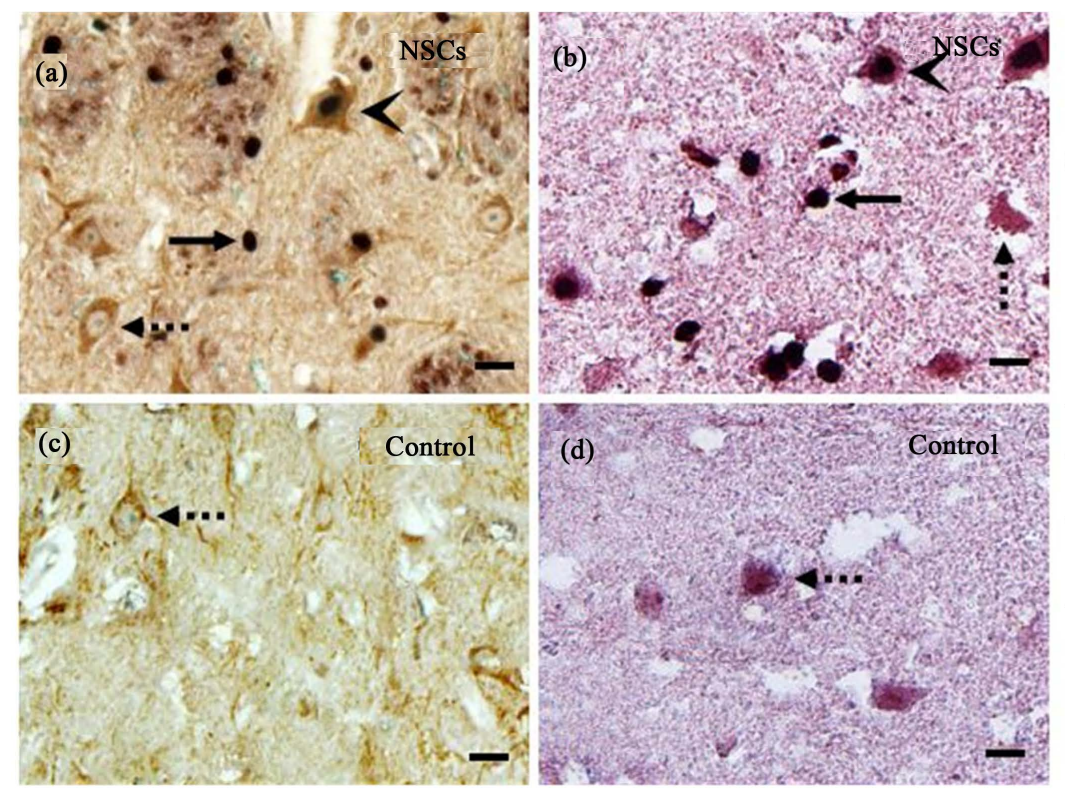

(e)

Figure 4. Immunohistochemical double staining of BrdU, MAP2 and GFAP positive cells in the corpus striatum. (a) $\mathrm{MAP}^{-} / \mathrm{BrdU}^{+}$cells (solid arrow), $\mathrm{MAP}^{+} / \mathrm{BrdU}^{-}$cells (dotted arrow) and $\mathrm{MAP}^{+} / \mathrm{BrdU}^{+}$cells (arrowhead) in NSCs group; (b) $\mathrm{GalC}^{-} / \mathrm{BrdU}^{+}$positive cells (solid arrow), $\mathrm{GalC}^{+} / \mathrm{BrdU}^{-}$cells (dotted arrow) and $\mathrm{GalC}^{+} / \mathrm{BrdU}^{+}$cells (arrowhead) in NSCs group; (c) $\mathrm{MAP2}^{+}$cells (dotted arrow) in control group; (d) $\mathrm{GalC}^{+}$cells (dotted arrow) in control group; (e) Quantitative analysis of the number of endogenous neurons and oligodendrocytes (mean $\pm \mathrm{SD}, \mathrm{n}=5$ ). ${ }^{*} P<0.05$ versus control group. Bar $=$ $25 \mu \mathrm{m}$. 
the expansion of encephalitogenic $\mathrm{T}$ cells, therefrom reducing immune cell mobilization from the periphery [12] [13]. Transplanted systemically NSCs entered perivascular CNS areas and subsequently induced apoptosis of blood-borne CNS-infiltrating encephalitogenic T cells [10] [14] [15]. Previous studies have showed that NSCs transplantation ameliorated the clinical symptoms and reduced tissue injury after EAE, which was related with reducing the number of perivascular infiltrates and of brain encephalitogenic $\mathrm{T}$ cells [8]. In this study, we showed a significant reduction in the number and size of inflammatory cell infiltrations in NSCs group than that in saline-injected group. These results indicate that NSCs effectively reduce infiltrating inflammatory cells, which may result in therapeutic efficacy on EAE.

Inflammatory environment inevitable results in axonal degeneration and demyelination, which is correlated with chronic disability and brain atrophy in advanced MS [16]. Transplanted NSCs generated new neurons and oligodendrocyte lineage cells to replace lost or degenerative cells, as a result markedly promoting axonal regeneration and decreasing the extent of demyelination [11] [17]. The present results showed transplanted NSCs differentiated into new neurons and oligodendrocytes, and promoted the proliferation of endogenous neurons and oligodendrocytes, thus probably promoting axonal regeneration and remyelination, leading to clinical recovery.

\section{Conclusion}

In summary, we show here that NSCs grafting significantly promotes functional recovery in EAE rats, reducing brain inflammatory infiltrations, improving density of myelin, and increasing repopulation of neurons and oligodendrocytes. These effects suggest a therapeutic efficiency of NSCs-based therapy on chronic EAE, thus probably providing a promising approach to therapy EAE or MS.

\section{References}

[1] Westerlind, H., Ramanujam, R., Uvehag, D., Kuja-Halkola, R., Boman, M., Bottai, M., et al. (2014) Modest Familial Risks for Multiple Sclerosis: A Registry-Based Study of the Population of Sweden. Brain, 137, 770-778. http://dx.doi.org/10.1093/brain/awt356

[2] Weisfeld-Adams, J.D., Katz Sand, I.B., Honce, J.M. and Lublin, F.D. (2015) Differential Diagnosis of Mendelian and Mitochondrial Disorders in Patients with Suspected Multiple Sclerosis. Brain, 138, 517-539. http://dx.doi.org/10.1093/brain/awu397

[3] Fletcher, J.M., Lalor, S.J., Sweeney, C.M., Tubridy, N. and Mills, K.H. (2010) T cells in Multiple Sclerosis and Experimental Autoimmune Encephalomyelitis. Clinical and Experimental Immunology, 162, 1-11. http://dx.doi.org/10.1111/j.1365-2249.2010.04143.x

[4] Furtado, G.C., Marcondes, M.C., Latkowski, J.A., Tsai, J., Wensky, A. and Lafaille, J.J. (2008) Swift Entry of MyelinSpecific T Lymphocytes into the Central Nervous System in Spontaneous Autoimmune Encephalomyelitis. Journal of Immunology, 181, 4648-4655. http://dx.doi.org/10.4049/jimmunol.181.7.4648

[5] Yang, J., Yan, Y., Ma, C.G., Kang, T., Zhang, N., Gran, B., et al. (2012) Accelerated and Enhanced Effect of CCR5Transduced Bone Marrow Neural Stem Cells on Autoimmune Encephalomyelitis. Acta Neuropathologica, 124, 491503. http://dx.doi.org/10.1007/s00401-012-0989-1

[6] Yang, J., Yan, Y., Xia, Y., Kang, T., Li, X., Ciric, B., et al. (2014) Neurotrophin 3 Transduction Augments Remyelinating and Immunomodulatory Capacity of Neural Stem Cells. Molecular Therapy, 22, 440-450. http://dx.doi.org/10.1038/mt.2013.241

[7] Grade, S., Bernardino, L. and Malva, J.O. (2013) Oligodendrogenesis from Neural Stem Cells: Perspectives for Remyelinating Strategies. International Journal of Developmental Neuroscience, 31, 692-700. http://dx.doi.org/10.1016/j.ijdevneu.2013.01.004

[8] Einstein, O., Grigoriadis, N., Mizrachi-Kol, R., Reinhartz, E., Polyzoidou, E., Lavon, I., et al. (2006) Transplanted Neural Precursor Cells Reduce Brain Inflammation to Attenuate Chronic Experimental Autoimmune Encephalomyelitis. Experimental Neurology, 198, 275-284. http://dx.doi.org/10.1016/j.expneurol.2005.11.007

[9] Chen, B., Gao, X.Q., Yang, C.X., Tan, S.K., Sun, Z.L., Yan, N.H., et al. (2009) Neuroprotective Effect of Grafting GDNF Gene-Modified Neural Stem Cells on Cerebral Ischemia in Rats. Brain Research, 1284, 1-11. http://dx.doi.org/10.1016/j.brainres.2009.05.100

[10] Pluchino, S., Zanotti, L., Ottoboni, B.L., Brambilla, E., Ottoboni, L., Salani, G., et al. (2005) Neurosphere-Derived Multipotent Precursors Promote Neuroprotection by an Immunomodulatory Mechanism. Nature, 436, 266-271. http://dx.doi.org/10.1038/nature03889 
[11] Pluchino, S., Quattrini, A., Brambilla, E., Gritti, A., Salani, G., Dina, G., et al. (2003) Injection of Adult Neurospheres Induces Recovery in a Chronic Model of Multiple Sclerosis. Nature, 422, 688-694. http://dx.doi.org/10.1038/nature01552

[12] Pluchino, S., Zanotti, L., Brambilla, E., Rovere-Querini P., Capobianco, A., Alfaro-Cervello, C., et al. (2009) Immune Regulatory Neural Stem/Precursor Cells Protect from Central Nervous Systemautoimmunity by Restraining Dendritic Cell Function. PLOS ONE, 4, e5959. http://dx.doi.org/10.1371/journal.pone.0005959

[13] Brambilla, R., Morton, P.D., Ashbaugh, J.J., Karmally, S., Lambertsen, K.L. and Bethea, J.R. (2014) Astrocytes Play a Key Role in EAE Pathophysiology by Orchestrating in the CNS the Inflammatory Response of Residen and Peripheral Immune Cells and by Suppressing Remyelination. GLIA, 62, 452-467. http://dx.doi.org/10.1002/glia.22616

[14] Aharonowiz, M., Einstein, O., Fainstein, N., Lassmann, H., Reubinoff, B. and Ben-Hur, T. (2008) Neuroprotective Effect of Transplanted Human Embryonic Stem Cell-Derived Neural Precursors in an Animal Model of Multiple Sclerosis. PLOS ONE, 3, e3145. http://dx.doi.org/10.1371/journal.pone.0003145

[15] Hackett, C., Knight, J. and Mao-Draayer, Y. (2014) Transplantation of Fas-Deficient or Wild-Type Neural Stem/Progenitor Cells (NPCs) Is Equally Efficient in Treating Experimental Autoimmune Encephalomyelitis (EAE). American Journal of Translational Research, 6, 119-128.

[16] Calza, L., Fernandez, M., Giuliani, A., Aloe, L. and Giardino, L. (2002) Thyroid Hormone Activates Oligodendrocyte Precursors and Increases a Myelin-Forming Protein and NGF Content in the Spinal Cord during Experimental Allergic Encephalomyelitis. Proceedings of the National Academy of Sciences of the United States of America, 99, 3258-3263. http://dx.doi.org/10.1073/pnas.052704499

[17] Rice, C.M., Kemp, K., Wilkins, A. and Scolding, N.J. (2013) Cell Therapy for Multiple Sclerosis: An Evolving Concept with Implications for Other Neurodegenerative Diseases. Lancet, 382, 1204-1213. http://dx.doi.org/10.1016/S0140-6736(13)61810-3 